\title{
Impact of COVID-19 on mental health research: is this the breaking point?
}

\author{
Oli Sparasci, Kamaldeep Bhui, Asit Biswas, Samuel Chamberlain, Bernadka Dubicka, \\ Robert Dudas, Saeed Farooq, Tamsin Ford, Nusrat Husain, lan Jones, Helen Killaspy, \\ William Lee, Anne Lingford-Hughes, Ciaran Mulholland, Judy Rubinsztein, \\ Rohit Shankar, Aditya Sharma, Lindsey Sinclair, James Stone and Allan Young
}

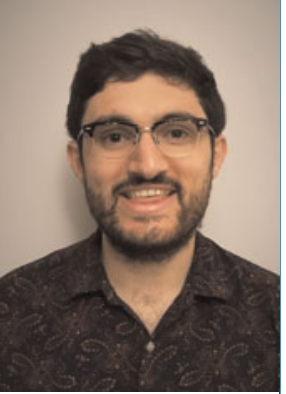

\section{Summary}

There are many structural problems facing the UK at present, from a weakened National Health Service to deeply ingrained inequality. These challenges extend through society to clinical practice and have an impact on current mental health research, which was in a perilous state even before the coronavirus pandemic hit. In this editorial, a group of psychiatric researchers who currently sit on the Academic Faculty of the Royal College of Psychiatrists and represent the breadth of research in mental health from across the UK discuss the challenges faced in academic mental health research. They reflect on the need for additional investment in the specialty and ask whether this is a turning point for the future of mental health research.

\section{Keywords}

Education and training; economics; clinical governance; costeffectiveness; supervision.

\section{Copyright and usage}

(C) The Author(s), 2022. Published by Cambridge University Press on behalf of the Royal College of Psychiatrists.
Oli Sparasci (pictured) is a higher trainee in Old Age Psychiatry in Oldham, his research at the University of Manchester focuses on novel diagnostic neuroimaging in dementia. Oli is the Psychiatric Trainees Committee Secretary for 2021/22. Kamaldeep Bhui is Professor of Psychiatry at the University of Oxford and an Honorary Consultant Psychiatrist. He is the Editor-in-Chief of the British Journal of Psychiatry. Asit Biswas is a Consultant Psychiatrist and Honorary Associate Professor. He is Vice-Chair of the RCPsych Intellectual Disability Faculty and has research interests in autism, intellectual disability and interventions for challenging behaviour. Samuel Chamberlain is based at the University of Southampton, his research focuses on the neurobiology and treatment of impulsive, compulsive and behaviourally addictive disorders. Bernadka Dubicka is a Consultant CAMHS Psychiatrist, Editor-in-Chief of the Journal of Child and Adolescent Mental Health and past chair of the faculty of Child and Adolescent Psychiatry. Robert Dudas is a Consultant Psychiatrist and Visiting Researcher at the University of Cambridge. His research interests include, dementia, borderline personality disorder, perinatal mood disorder, values-based practice and the medical humanities. Tamsin Ford is Professor of Child and Adolescent Psychiatn. Her research focuses on the organisation, delivery and effectiveness of services and interventions for children and young people's mental health. Nusrat Husain is Professor of Psychiatry and Honorary Consultant Psychiatrist. His research focuses on global mental health and cultural psychiatry. Ian Jones is Professor of Psychiatry and Honorary Consultant Psychiatrist. His research focuses on bipolar spectrum disorder, in particula around the time of childbirth. Helen Killaspy leads national and international research programmes focusing on improving the quality of care and outcomes for people with complex psychosis. William Lee is a Consultant Liaison Psychiatrist at Cornwall Partnership NHS Foundation Trust and Honorary Senior Clinical Lecturer and the University of Exeter. Anne Lingford-Hughes is Professor of Addiction Biology and Head, Division of Psychiatry at Imperial College and Honorary Consultant Psychiatrist at Central North West London NHS Foundation Trust, UK. Her work focuses on understanding the neurobiology of addiction, primarily alcohol and opiate, to improve treatment. Ciaran Mulholland is a Senior Lecturer at Queens University Belfast and a Consultant Psychiatrist. His research interests are broad and include first-episode psychosis and the impact of the 'Troubles' on mental health. Judy Rubinsztein is a Consultant in Old Age Psychiatry, her research interests at the University of Cambridge are in crisis teams, bipolar disorder in later life and medical education. Rohit Shankar is Professor in Neuropsychiatry, Consultant in Adult Developmental Neuropsychiatry, he is Clinical Director of Adult Learning Disabilities Services at Cornwall Foundation Trust. Aditya Sharma is a Clinical Senior Lecturer and Honorary Consultant in Child and Adolescent Psychiatry, his research focuses on bipolar disorder in younger people. Lindsey Sinclair is a Clinical Research Fellow in Psychiatry at the University of Bristol. She is the current Financial Officer of the RCPsych Academic Faculty. James Stone is a Reader in Psychiatry at Brighton and Sussex Medical School and Honorary Consultant in Liaison Psychiatry at Sussex Partnership NHS Foundation Trust. Allan Young is Chair of Mood Disorders and Director of the Centre for Affective Disorders in the Department of Psychological Medicine at the Institute of Psychiatry, Psychology and Neuroscience at King's College London.

\section{COVID-19}

The coronavirus disease 2019 (COVID-19) pandemic has exposed organisational and societal vulnerabilities to new infectious diseases and highlighted the socioeconomic consequences of lockdown, underpinned by inequalities in access to power and resources. The National Health Service (NHS), social care, public transport, schools, universities and businesses have been tested up to, and beyond, breaking point, resulting in job losses, service failure and poverty. The impact on vulnerable groups, such as those in ethnic minorities, elderly people in care homes and people with intellectual disabilities (also known as learning disabilities in UK health services), was stark in terms of excess mortality. We as a society are still reeling from the shock, and it is unlikely that we will fully understand the full impact of the pandemic for many years.

In this article, we highlight the significant impact that the pandemic has had on mental health research and discuss the consequences of this on clinical practice, professional education and the training of future psychiatrists. Research is a core element of health service design globally and must be viewed as central to providing safe, effective and adaptable mental healthcare, which is increasingly important during times of great international change. Thus, we aim to make the case that mental health research serves a purpose beyond the purely scientific endeavour of intellectual discovery and at present is underfunded when considering the wider burden of mental ill health

As we emerge from the acute phase of the pandemic, we can start to appraise other critically vulnerable social and institutional structures and practices. We need an improved way of delivering healthcare. Arguably the past 18 months have seen a greater focus on the mental health of individuals and populations than at any time in our history. The public discourse has been dominated by predictions of dire mental health consequences from politicians, journalists and commentators. The cause of any increase in mental health problems is variously ascribed; sometimes to the pandemic itself, sometimes to the unintended consequences of the lockdown, and sometimes to both. What is clear, is that the predicted rise of mental ill health has not yet fully emerged, ${ }^{1}$ although the most vulnerable members of our society have been disproportionately affected, particularly those living in poverty, with pre-existing mental or physical health needs and those at both ends of the age spectrum. Furthermore, the full mental health impact of long COVID remains unclear and is likely to be significant. 


\section{Training}

Although the impact of the pandemic on population mental health remains uncertain, the influence on psychiatric practice, training and research is clearer. The detrimental effects of COVID on the delivery of mental healthcare have been profound, but have been well documented elsewhere. Mental health research, which was already in a precarious position, has been further challenged. Significant gaps remained in our understanding of mental illness prior to the pandemic, during which research progress has slowed. The start of the pandemic saw the redeployment of psychiatry trainees and consultants to COVID-related roles, leading to significant disruption to both mental health services and to individuals' work and personal lives. As a result, services were often sustained with minimum staffing levels, exacerbated by requirements for short- and long-term self-isolation. To date, clinical activities have not returned to pre-pandemic levels. Trainee progression has also been affected. For trainees in research, many have been left trying to resolve the challenges of insufficient time and funding. Others returned to full-time clinical care during the pandemic, and are now facing major barriers (including filling rota gaps owing to failures of planning rather than the pandemic) when attempting to return to research. This has in large part been because of organisational inertia within the healthcare research structure during much of the pandemic to date.

Academic psychiatry was already in difficulty and these disruptions to training and research are likely to exacerbate chronic problems with careers in psychiatric research. Major universities have been seeking high-performing professors and their associated grant income with little support for a broader range of earlycareer psychiatrists to obtain research training. The lack of lecturer and senior lecturer posts, and the closure of psychiatry departments in some universities is continuing, which has seen a paucity of earlycareer academics emerging. Time-limited fellowships have gone some way to ameliorating this, however, this should not be seen as a replacement for substantive research posts. Thus, our ability to meet current and future research priorities is further weakened. Only through research, linked with quality improvement and implementation science working synergistically, can we hope to build a safer, more effective and humane care system, one that is robust to crises and not the immediate target for redeployment or resource cuts at such times.

\section{Services}

The economic impact of the pandemic meant research to improve integrated and high-quality care was again undermined. In addition, despite some NHS trusts supporting trainees and consultants to include research as a core part of their job plans, such good practice is sadly far from universal. The problem of lack of protected research time existed prior to COVID, while during the pandemic and its aftermath, more psychiatrists are unable to pursue research, despite the pressing need for data to support service evaluation and quality improvement as well as intervention trials. Many sessions for research that were removed at the height of the pandemic have yet to be restored. Frequently, short-term contracts for university staff were not extended despite the continuing, if not increased demands for their work, especially in teaching and supervision. This was seen under the guise of managing short-term finances and as a necessary response to the crisis.

Mental health research has long been underfunded, but since the start of the pandemic, calls have been cancelled and funds have been withdrawn. ${ }^{2}$ Many ongoing grants have not been extended despite COVID-19-related delays, and a $£ 120$ million cut in funding by UK Research and Innovation has been implemented. The Medical Research Council commented on 'mental health' only twice in their review of medical research funding during the pandemic. ${ }^{3}$ Research commissioners have diverted funds to COVID, funding a small number of large grants at relatively few institutions for short periods of time, in the hope that research can be completed in less time than is necessary.

The rapid vaccination development programme has produced a model of academic advancement that is not easily transferable to the need for better interventions in mental healthcare, nor for developing a future cohort of researchers working in mental health and related interdisciplinary areas of scholarship. Furthermore, the focus on COVID-19 has led to a large volume of papers published, many of which are poor quality, with several high-profile retractions. ${ }^{4}$ Mental health funding for COVID-19 research has been limited given the vast scope of the problem and the need to rigorously pursue synergistic efforts across disciplines to adequately capture and mitigate the impact on mental health. In parallel, the World Health Organization has noted profound disruption to clinical mental health services during the pandemic across the globe, which will itself impede clinically focused research opportunities. ${ }^{5}$

\section{Academic Psychiatry}

The ongoing pressures of COVID-19 along with the low priority of mental health research in the UK are likely to have a further impact on the long-term viability of UK academic psychiatry. We have reached a point where reversal of these long-term trends will be difficult and full attention to reinvigorating mental health research must be given to prevent its further deterioration. Reduced time and funding for research will further worsen career progression and the pre-existing failure to recruit sufficiently diverse researchers will continue to highlight structural problems within much of the current mental health research output.

It is essential that mental health should be allocated an equitable system of research funding, training and practice that reflects a reasonable work-life balance and does not promote inequalities. Potential for discrimination and racism in universities is well recognised and requires positive systems-wide actions. More provision should be made to allow clinical psychiatrists to also be involved with research - this could be through protected research time, which may help to address recruitment difficulties to many consultant psychiatry posts. Our experience with NHS trusts who do include research as part of advertised job proposals is that such trusts are likely to attract more job candidates as well as being more likely to retain staff in the long term. Similarly, there is evidence that research-active trusts have better clinical outcomes. It is clear that there are systemic changes that need to be made to ensure the future of high-quality research as we move beyond the acute phase of the pandemic.

The Academic Faculty of the Royal College of Psychiatrists call upon our fellow researchers, practitioners and policymakers to address parity in resources and to recognise the centrality of mental healthcare and thus mental health research to the global post pandemic recovery.

Opportunities arising because of increasing global connectivity could be harnessed to develop and implement mental health research more meaningfully, particularly as a large burden of mental health matters sit in low- and middle-income countries. The pandemic could be a lever for positive change to bring research communities across continents and disciplines together. With these changes, we might be able to bring academic psychiatry successes back in line with other world-class research from the UK.

Integrating clinical work and academic practice will require a change to existing structures, where the NHS and universities operate to divergent priorities, interests and business models. Without a progressive, integrated approach, in response to 
evident weaknesses unearthed by COVID-19, we might be mourning academic psychiatry as the specialty that no one knew they needed until it was gone.

Oli Sparasci $\mathbb{D}$, Department of Psychiatry, Pennine Care NHS Foundation Trust, UK Kamaldeep Bhui $(\mathbb{D}$, Department of Psychiatry \& Nuffield Department of Primary Care Health Sciences, University of Oxford, UK; Asit Biswas (D), Learning Disabilities Department, Leicestershire Partnership NHS Trust, UK; Samuel Chamberlain (D.) Department of Psychiatry, Faculty of Medicine, University of Southampton, UK Bernadka Dubicka (iD), The University of Manchester and Pennine Care NHS Foundation Trust, UK; Robert Dudas (D), Department of Psychiatry, University of Cambridge, UK; Saeed Farooq $(\mathbb{D}$, Keele University, UK and Midlands Partnership NHS Foundation Trust, UK; Tamsin Ford (D), University of Cambridge, UK and Cambridge and Peterborough NHS Foundation Trust, UK; Nusrat Husain (D), The University of Manchester, UK and Lancashire Care NHS Foundation Trust, UK; Ian Jones (iD), National Centre for Mental Health, Cardiff University, UK; Helen Killaspy (D), Division of Psychiatry, University College London, UK; William Lee (D), University of Exeter, UK and Cornwall Partnership NHS Foundation Trust, UK; Anne Lingford-Hughes (D), Division of Psychiatry, Imperial college London, UK; Ciaran Mulholland $\mathbb{D}$, Queen's University Belfast School of Medicine Dentistry and Biomedical Sciences, UK and Northern Health and Social Care Trust, UK; Judy Rubinsztein (D), Cambridgeshire and Peterborough NHS Foundation Trust, UK and Department of Psychiatry, University of Cambridge, UK; Rohit Shankar (1) Cornwall Intellectual Disability Equitable Research, University of Plymouth Peninsula School of Medicine, UK; Aditya Sharma (D), Translational and Clinical Research Institute Newcastle University, UK; Lindsey Sinclair (D), Bristol Medical School, University of Bristol, UK; James Stone $\mathbb{D}$, Brighton and Sussex Medical School, University of Sussex UK; Allan Young (D), Department of Psychological Medicine, Institute of Psychiatry, Psychology and Neuroscience, King's College London, UK

Correspondence: James Stone. Email: j.stone@bsms.ac.uk

First received 12 Aug 2021, final revision 30 Nov 2021, accepted 21 Dec 2021

\section{Author contribution}

All authors contributed to the conception and design. J.S. and O.S. led the writing and drafting consecutive versions with all authors contributing additional edits, review, and suggestions on revisions.

\section{Funding}

This research received no specific grant or assistance from any funding agency, institution, commercial or not-for-profit organisation.

\section{Declarations of interest}

K.B. is the Editor-in-Chief of the British Journal of Psychiatry. S.C. is funded by the Wellcome Trust, he has received honoraria from Elsevier and previously consulted for promentis. B.D. receives funding from the NIHR HTA programme. T.F. receives funding from UKRI to track the impact of COVID-19 on the mental health of children and young people and consults for Place2Be N.H is the chair of board of trustees of Manchester Global Foundation a Charitable Incorporated Organisation registered in England and Wales. He has received honorarium and travel grants from various pharmaceutical industries. He is an NIHR senior investigator. I.J. receives funding from Health and Care Research Wales to study the impact of COVID-19 on people with lived experience of mental illness. A.L.-H. is a Handling Editor of BJPsych and was the immediate past chair of the Academic Faculty of the Royal College of Psychiatrists; she has received research support and honoraria from Lundbeck, GSK and Alcarelle paid consultancy to Silence and unpaid consultancy to Astrazeneca, Opiant, Britannia Pharmaceuticals a, UCB, Eisai, Veriton Pharma, Bial, Averelle and GW pharmaceuticals outside the submitted work. L.S. Is funded by a Junior Fellowship from the Alzheimer's Society. J.S. has been principal investigator or chief investigator on studies sponsored by Takeda, Janssen and Lundbeck PIc. He has attended an Investigators' meeting run by Allergan Plc. A.Y. is deputy Editor of BJPsych Open, has received payment for lectures or advisory work from: Astrazeneca, Eli Lilly, Lundbeck, Sunovion, Servier, LivaNova, Janssen, Allegan, Bionomics, Sumitomo Dainippon Pharma, COMPASS. Is a consultant to Johnson \& Johnson and LivaNova. Received honoraria for attending advisory boards and presenting talks at meetings organised by LivaNova. Principal investigator in the Restore-Life VNS registry study funded by LivaNova. Principal investigator on ESKETINTRD3004. Is the principal investigator on 'The Effects of Psilocybin on Cognitive Function in Healthy Participants.' Is principal investigator on 'The Safety and Efficacy of Psilocybin in Participants with Treatment-Resistant Depression (P-TRD).' UK chief Enestigator for NOvartis MDD Study MU1821A12201. Grant funding (past and present): NIMH (USA); CIHR (Canada); NARSAD (USA); Stanley Medical Research Institute (USA); MRC (UK): Wellcome Trust (UK); Royal College of Physicians (Edin); BMA (UK); UBC-VGH Foundation (Canada); WEDC (Canada); CCS Depression Research Fund (Canada); MSFHR (Canada); NIHR (UK). Janssen (UK). No shareholdings in pharmaceutical companies. W.L. is deputy editor of the British Journal of Psychiatry. A.B., R.D., S.F., H.K., C.M., J.R., A.S. and O.S. have no competing interests to declare.

\section{References}

1 Shevlin M, McBride O, Murphy J, Miller JG, Hartman TK, Levita L, et al. Anxiety, depression, traumatic stress and COVID-19-related anxiety in the UK general population during the COVID-19 pandemic. BJPsych Open 2020; 6: e125.

2 Alzheimer's Research UK. One in Three Dementia Scientists Consider Leaving Research Due to COVID-19. Alzheimer's Research UK, 2020 (https://www. alzheimersresearchuk.org/one-in-three-dementia-scientists-consider-leavingresearch-covid-19/)

3 Chinnery PF, Pearce JJ, Kinsey AM, Jenkinson JM, Wells G, Watt FM. How COVID-19 has changed medical research funding. Interface Focus 2021; 11 20210025.

4 Retraction Watch. Retracted Coronavirus (COVID-19) Papers. Retraction Watch, 2020 (https://retractionwatch.com/retracted-coronavirus-covid-19papers/).

5 World Health Organization. The Impact of COVID-19 on Mental, Neurological and Substance use Services. WHO, 2020 (https://www. who.int/publicationsdetail-redirect/978924012455). 